Espacio, Tiempo y Forma, Serie II, Historia Antigua, t. 23, 2010, págs. 217-230

\title{
Las relaciones interpersonales entre los soldados de la guarnición de Roma
}

\author{
Interpersonal relations between soldiers of the garrison of Rome
}

\author{
Héctor Ceñal MartíneZ*
}

\begin{abstract}
RESUMEN
La finalidad de este artículo es la de realizar una aproximación a las relaciones personales, afectivas o incluso de parentesco que unían entre sí a los distintos soldados que componían la guarnición de la Urbe. Se obviarán aquellas vinculaciones debidas a motivos políticos o protocolarios, o las relaciones

de soldados pertenecientes a la misma cohorte. Es por ello que las dedicaciones comunales realizadas en honor del emperador de forma oficial y no a título personal al quedado excluidas de este análisis, al igual que los latercula. Nuestro interés radica en establecer las relaciones entre soldados que no poseían un contacto directo y diario.

PALABRAS CLAVE: Amistad, parentesco, relaciones personales, heredero, cohortes pretorianas, cohortes urbanas, vigiles, singulares.
\end{abstract}

\section{ABSTRACT}

The aim of this article is to present a general idea of the interpersonal relations, affective and familiar, that connected the soldiers of the urban garrison. Ties owing to political or ceremonial reasons will be left out, as well as relations between soldiers of the same cohort. For this reason official dedications made in common in the Emperor's honour, and not personally, have been excluded from this analysis, and likewise for the latercula. Our interest is focused on establishing the relations between soldiers that did not have daily and direct contact.

\section{KEY WORDS:}

Friendship, relationship, personal relations, heir, praetorian cohorts, urban cohorts, vigiles, singulares.

* Facultad de letras (Ha Antigua). Universidad de Oviedo. C/ Alfonso Martínez s.n. 33011. septimani@ hotmail.com 


\section{RELACIONES INTERPERSONALES DENTRO DEL PRETORIO.}

El primer tipo de confraternización que vamos a estudiar existe únicamente dentro del pretorio. Los soldados pretorianos aún perteneciendo a distintas cohortes establecen relaciones de amistad importantes entre sí, hecho que no sucede con soldados de otras unidades militares aún perteneciendo a cohortes de la misma clase, como pudieran ser los urbaniciani o los vigiles.

Éste es así mismo el grupo que mayor número de ejemplos nos proporciona (15) en un abanico que va desde el gobierno de Vespasiano hasta mediados del siglo III d. C.

a) Relación entre difunto (a quien se dedica el epígrafe) y los dedicantes del epígrafe (normalmente herederos):

En primer lugar tenemos aquellos casos, de los que desconocemos cómo surgió la amistad que unió en vida a ambos soldados':

- CIL XI 6348 (50-100 d. C.): Quintus Seienus Quinctianus de la cohorte VII Pretoriana, de cuyo epígrafe se encargó Publius Aelius Mucianus de la cohorte I Pretoriana.

- CIL VI 3893 = CIL VI 32687 (100-180 d. C.): Sextus Lullius Aponianus, euocatus de la cohorte $\mathrm{V}$ Pretoriana, y Lucius Aponius Apollinaris de la cohorte $\mathrm{X}$ Pretoriana.

- CIL VI 2700 (120-180 d. C.): Caius Baetus Maximus de la cohorte IX Pretoriana y Marcus Granius Serenus de la cohorte V Pretoriana.

- CIL VI 2433 (213-227 d. C.): Iulius Surus de la cohorte I Pretoriana y Aurelius Diogenes de la cohorte VII Pretoriana.

- AE 198350 (época de Caracalla o de Heliogábalo): Lucius Aurelius Diza de la cohorte VI Pretoriana antoniniana pía vengadora y Aurelius Longinus de la cohorte X Pretoriana antoniniana.

- CIL VI 2523 (200-250 d. C.): Quintus Arruntius Hospes de la cohorte IIII Pretoriana y Aelius Maximus de la cohorte VII Pretoriana.

- CIL VI 2695 (200-250 d. C.): Marcus Aurelius lanuarius de la cohorte VIIII Pretoriana y Aurelius Verenus Secundus, centurión de la cohorte II Pretoriana.

- CIL VI 2752 (200-250 d. C.): Marcus Simplicinius Superinus de la cohorte X Pretoriana pía vengadora y sus dos herederos, Caius Valerius Valens y

Ricci, C., 1999: 30, señala que todos los soldados enterrados por sus herederos habían fallecido fuera del lugar de nacimiento, especialmente sensible debía de ser esta situación para los soldados pretorianos extraitálicos cuando fallecía un conmilitón y convecino. 
Marcus Drusinius Lupulus, ambos de la cohorte VI Pretoriana pía vengadora y de la misma centuria.

- CIL VI 2742 (200-270 d. C.): Diogenes Gaius de la cohorte X Pretoriana y Aurelius Herodes de la cohorte VII Pretoriana. El dedicante se define como hermano del fallecido lo que refleja una fuerte amistad entre ambos.

En algunos de los casos sabemos que la razón por la que fallecido y heredero habían entablado amistad era que ambos compartían el mismo origen geográfico. Poseemos diversos ejemplos:

- CIL VI 2520 (100-150 d. C.): Titus Aelius Marcellus de la cohorte IIII Pretoriana y Titus Flavius Petronianus de la cohorte II Pretoriana, ambos procedentes de Doberus.

- CIL VI 2583 = CIL XI 15610 (en torno al año 150 d. C.): Publius Mestrius Maximus de la cohorte V Pretoriana y Publius Acconius Crescens de la cohorte IIII Pretoriana.

- CIL VI 2732 (siglo III d. C.): Aurelius Burus de la cohorte X Pretoriana y Aurelius Diszarai de la cohorte VIII Pretoriana.

- AE 1924107 (120-180 d. C.): Publius Aelius Respectus de la cohorte VI Pretoriana y Publius Aelius Tutor de la cohorte VIII Pretoriana, ambos en el mismo epígrafe siendo el dedicante Publius Aelius Tutor de la cohorte IX Pretoriana ${ }^{2}$.

En otros casos, fallecido y heredero estaban emparentados, como se deduce del praenomen y del nomen común. Normalmente los familiares servían en la misma cohorte, pero existen algunas excepciones:

- AE 1934139 (anterior al año 79 d. C.): Lucius Terentius Secundus de la cohorte VI Pretoriana y Caius Terentius Fronto de la cohorte III Pretoriana, hermanos.

- AE 191649 (100-150 d. C.): Lucius Aurelius Dignus de la cohorte V Pretoriana y Lucius Aurelius Dionysianus de la cohorte I Pretoriana.

b) Relación entre los dedicantes de un voto:

- CIL VI 478 (siglo II d. C.): Titus Livius Successus de la cohorte I Pretoriana y Lucius Clarennius Verus de la cohorte V Pretoriana, sin hallarse vinculados por parentesco o un origen común, dedican juntos un voto a Marte, único ejemplo del siglo II d. C.

2 El epígrafe está en primer lugar dedicado al difunto Publius Aelius Finitus de la cohorte IX Pretoriana, hermano del dedicante, siendo incluido después ambos conciudadanos. Dada la coincidencia de nomina es posible que exista algún parentesco, posiblemente lejano, entre ambos. 
- CIL VI 2385b $19=$ CIL VI 2385b $24=$ CIL VI 2388a $1=$ CIL VI 2388a $2=$ CIL VI 2388a 3 = CIL VI 2388a $4=$ CIL VI $3797=$ CIL VI $3798=$ CIL VI $31491=$ CIL VI 32538 (213 d. C.): Marcus Venuleius Felix y Titus... Iuvencatus, centuriones de la cohorte VII Pretoriana antoniniana pía vengadora; Caius... Claudianus, centurión de la cohorte VI Pretoriana antoniniana pía vengadora; Calpurnius Cerialis, centurión de la cohorte II Pretoriana antoniniana pía vengadora y Maximianus, centurión de la cohorte IIII Pretoriana antoniniana pía vengadora, quienes honran al emperador Caracalla. Todos ellos procedían de distintos lugares, por lo que es evidente que se encontraban vinculados por cuestiones de rango.

- CIL VI 2799 = CIL VI 32543 = ILS 2094 (227 d. C.): Marcus Aurelius Diza, Marcus Aurelius Diza ${ }^{3}$ y Marcus Aurelius Crescens de la cohorte I Pretoriana; Marcus Aurelius Martinus, Marcus Aurelius Bitus, Marcus Aurelius Maximus y Marcus Aurelius Maximus de la cohorte II Pretoriana; Marcus Aurelius Vitalis, Marcus Aurelius Apollodorus y Marcus Aurelius Vitalis de la cohorte III Pretoriana; Caius Valerius Valens y Marcus Aurelius Cassius de la cohorte IIII Pretoriana; Marcus Aurelius Diogenes de la cohorte VII Pretoriana; Marcus Aurelius Diza y Marcus Aurelius Diza de la cohorte VIII Pretoriana; Marcus Aurelius Chrestus de la cohorte VIIII Pretoriana y finalmente Marcus Aurelius Artila, Marcus Aurelius Ota..., Marcus Aurelius Bhitus y Aurelius Mucianus de la cohorte $X$ Pretoriana. Todos ellos eran naturales de Philippopolis y dedican el voto a Esculapio Zimidrenus.

- Iulius lustus, de la cohorte I Pretoriana pía vengadora, y Valens y Firmius Maternianus, de la cohorte X Pretoriana pía vengadora, naturales de Augusta Viromandorum. Estos soldados dedicaron dos votos distintos, el primero en época de Gordiano III a los dioses santos tutelares, Júpiter óptimo máximo, Apolo, Mercurio, Diana, Hércules y Marte (CIL VI $2822=\mathrm{CIL}$ VI $3902=\mathrm{CIL}$ VI 3903 = CIL VI 32550a-b = AE 1894 18); y el segundo en época de Filipo I a Júpiter óptimo máximo, Marte, Némesis, el Sol, la Victoria y todos los dioses de la patria (CIL VI $2821=$ CIL VI $32551=$ ILS 2096). Dado que se trata de los dos únicos soldados pretorianos con esta procedencia, es posible que ambos se conociesen con anterioridad a su ingreso en el pretorio.

- CIL VI 2831= CIL VI 2852 = CIL VI 32557 (gobierno de Trajano Decio): : Bomanus de cohorte pretoriana desconocida, Datuorius y Maximianus de la cohorte V Pretoriana, y dos soldados anónimos, uno de la cohorte VII y otro de la cohorte $\mathrm{X}$, todos ellos ciudadanos cotinii, dedican juntos un voto a la Fortuna, a Apolo y a la Victoria.

- CIL VI 2807 = CIL VI 32582 = ILS 4068 (siglo III d. C.): Flabius Proclianus de la cohorte III Pretoriana, Claudius Mucianus y Aurelius Valerianus, ambos de la cohorte V Pretoriana y Valerius Maximus de la cohorte X Pretoriana, todos ellos naturales de Agatapara, dedican juntos un voto a Hero Briganitius.

\footnotetext{
${ }^{3}$ En este epígrafe son frecuentes los fenómenos de homonimia.
} 
- CIL VI 32571 (siglo III d. C.): Un soldado desconocido de la cohorte II Pretoriana, otro de la cohorte VIII, Tacens y Mucatra de la cohorte VIIII, dedican juntos un voto a Apolo de Raimullovluzolum.

- CIL VI 2845 = CIL VI 32605 (siglo III d. C.): Aurelius Aurelianus de la cohorte II Pretoriana y Epicadus de la cohorte I Pretoriana, ambos de Dardania.

\section{RELACIONES INTERPERSONALES ENTRE LOS PRETORIANOS Y LOS URBANICIANI}

Apenas 7 ejemplos representan a este segundo grupo, a pesar de que al menos hasta época de Commodo los urbaniciani se encontraban acantonados dentro de los Castra Praetoria, por lo que cabría esperar la existencia de relaciones más fluidas. El marco temporal de los epígrafes nos lleva de Nerón hasta principios del siglo III d. C., interrumpiéndose en todo caso los vínculos personales entre pretorianos y urbaniciani desde el gobierno de Septimio Severo. Esto vendría a corroborar la idea de que los urbaniciani fueron trasladados al Castra Urbana durante el gobierno de Commodo 4 .

De nuevo, en la mayoría de los casos los soldados ocupan los papeles de difunto y heredero / dedicante del epígrafe, ignorando de donde provenía su amistad. Existen únicamente dos ejemplos de esto. El primero, de fecha indeterminada en los siglos I ó II d. C., es el de lulius Amator de la cohorte III Pretoriana y lunius Felix de la cohorte X Urbana (AE 1969/70 172 = Inscrlt III 1, no 132), quienes no estaban unidos por parentesco.

El segundo, del siglo II d. C., es el de Caius Dicius Sabinus de la cohorte VIII Pretoriana y Marcus Bellicus Valens de la cohorte XI Urbana, quienes tras fallecer fueron honrados en un epígrafe común por Caius Deccius Maximianus, beneficiario del prefecto urbano $(\mathrm{CIL}$ VI $2680=\mathrm{CIL}$ VI 32658). Probablemente Caius Deccius Maximianus estaba emparentado con Caius Dicius Sabinus.

En el resto de los casos hay un vínculo de sangre, que une a dedicado y dedicante, $\mathrm{o}$ incluso a dos familiares difuntos:

- AE 1981 388: Caius Samius Crescens de la cohorte XII Urbana, difunto, y su heredero y hermano Samius Fortis de la cohorte VIII Pretoriana.

- AE 198352 (entre los gobiernos de Claudio y Nerón): Lucius Licinius Pudens de la cohorte IIII Pretoriana y Sextus Licinius Mansuetus de la cohorte XV Urbana, fallecidos ambos y mencionados en el mismo epígrafe, eran hermanos.

- CIL VI 2714 (150-190 d. C): Titus Lucconius Quartinus de la cohorte IX Pretoriana y Titus Lucconius Paternus de la cohorte XII Urbana, hermanos honrados por su madre.

\footnotetext{
4 Sablayrolles, R. 2001: 140.
} 
- CIL X 1761 (entre el siglo II d. C. y la llegada al poder de Septimio Severo): Lucius Valerius Fronto de la cohorte X Pretoriana y Marcus Valerius Fronto de la cohorte XIIII Urbana, hermanos honrados por su madre.

- CIL VI 2579 (211-217 d. C.): Caius Iulius Iulianus, veterano de la cohorte V Pretoriana pía vengadora, cuyo epígrafe está dedicado por Caius lulius Glaus, soldado de la legión II Parthica antoniniana pía fiel feliz eterna, y por Caius Iulius de la cohorte XII Urbana, quienes eran sus hermanos.

\section{RELACIONES INTERPERSONALES ENTRE LOS PRETORIANOS Y LOS EQUITES SINGULARES AUGUSTI}

Las relaciones personales entre los soldados del pretorio y los singulares parecen haber sido más frecuentes que con el resto de unidades militares presentes en la ciudad de Roma, ya que existen 14 inscripciones representativas de ello. Todas ellas fechadas en el siglo III d. C.

En este caso todas las vinculaciones entre pretorianos y singulares se sitúan en la línea difunto y heredero / dedicante del epígrafe, sin que conozcamos cómo surgió la amistad que les unía. Encontramos los siguientes casos:

Denkm 744 = AE 1989 78: Caius Iulius Victor de la cohorte I Pretoriana y Caius Valerius Lepidinus, duplicarius de los jinetes singulares del emperador.

- AE 2004319 (bajo el gobierno de Heliogábalo o de Alejandro Severo): Marcus Selvinius lustinus de la cohorte VII Pretoriana pía vengadora y Veracius Provincialis, jinete singularis.

- CIL XIV 2952 = Denkm 746 (fin del siglo II-primera mitad del siglo III d. C.): Titus Flavius Paternus de la cohorte X Pretoriana y Publius Aelius Candidus, jinete singularis.

- AE 1993165 (siglo III d. C.): Jinete singularis desconocido y un soldado desconocido de la cohorte II Pretoriana.

- CIL VI 2486 = Denmk 749 (siglo III d. C.): Aurelius lobinus de la cohorte III Pretoriana y Aurelius Martinus, jinete singulares.

- CIL VI 3241 = Denmk 606 (siglo III d. C.): Candidius Valentinus, jinete del numerus de los singulares, y sus dos herederos: Arrius Latianus de la cohorte IIII Pretoriana y Flavius Vitalis de la cohorte X Pretoriana.

- CIL VI 3239a = Denmk 641 (siglo III d. C.): Marcus Aurelius..., jinete singularis de nuestro señor y un soldado pretoriano desconocido.

- CIL VI 2457 = CIL XI 3845 = Denkm 748 (siglo III d. C.): Aelius Cogitatus, veterano de nuestros emperadores, cuyo epígrafe esta dedicado por varios amigos, entre ellos Lollius Constantinus de la cohorte II Pretoriana y Ulpius Marcellianus, jinete singularis de nuestros emperadores. 
- CIL VI 3277 = Denmk 736 (siglo III d. C.): Caius Marius Gemellinus, veterano de los jinetes singulares de nuestro emperador, y Aurelius Emeritus, soldado llamado de nuevo de nuestro emperador.

- CIL VI 3214 = Denkm 108 (siglo III d. C.): Titus Aurelius Aelius Maximus, signifer de los jinetes singulares de nuestro emperador, y Titus Flavius Firmus, soldado llamado de nuevo del emperador.

En otros casos, fallecido y heredero estaban emparentados, siendo no obstante un fenómeno infrecuente:

- ILS $9069=$ AE 189433 = Denkm 747 (161-260 d. C.): Caius Vedinacus Dexter, optio de la cohorte II Pretoriana, y Marcus Iulius Secundus, jinete de los emperadores $\mathrm{y}$, al parecer, padrastro de aquél.

- CIL VI 32680 = CIL X 3582 = Denmk 750 (200-250 d. C.): Marcus Aurelius Dasius de la cohorte V pretoriana pía vengadora y Marcus Aurelius Candidus, jinete singularis de nuestro señor.

- AE 195479 = Denmk 530 (siglo III d. C.): Natalinius Natalianus, decurión del numerus de los jinetes singulares de nuestro señor y Natalinius... de la cohorte II Pretoriana, probablemente un hijo o un nieto.

- AE 198349 = Denmk 608 (siglo III d. C.): Aurelius Daicos, jinete singulares, y Aurelius Daicon de la cohorte VIII Pretoriana, hermano del anterior.

\section{RELACIONES INTERPERSONALES ENTRE LOS PRETORIANOS Y SOLDADOS DE LA LEGIÓN II PARTHICA}

Los soldados de la legión II Parthica no se encuentran acantonados en Roma sino en Alba, pero la proximidad con la Urbe, apenas 15 kilómetros, la participación de ambas tropas en las mismas campañas hizo posible que entre pretorianos y albanoi se estableciesen diversas simpatías.

La relación entre el difunto y heredero o los herederos se encuentra como siempre presente:

- CIL VI 3402 (siglo III d. C.): Marcus Aurelius... de la legión II Parthica y Aurelius Ve... de cohorte pretoriana desconocida.

- CIL XIII 8516 (siglo III d. C.): Victor, adiutor del tabulario del princeps de la legión II Parthica, y Severus, beneficiario del prefecto del pretorio. albanoi:

Existe además un caso en el que existe parentesco entre los pretorianos y los

- CIL VI 3894 = CIL VI 32690 (siglo III d. C.): Aurelius Pius de la cohorte VI Pretoriana, cuyo epígrafe está dedicado por Aurelius Pompeianus, hermano y soldado de la legión II Parthica, y por Valerius Valens, primo hermano por parte de madre y soldado de la cohorte VI Pretoriana. 


\section{RELACIONES INTERPERSONALES ENTRE LOS PRETORIANOS Y OTRAS TROPAS PRESENTES EN LA URBE}

Los vínculos afectivos o de parentesco son mucho más infrecuentes entre los pretorianos y el resto de soldados establecidos en la Urbe. De hecho, sólo cuatro ejemplos integran este grupo:

- CIL XVI 10 = AE 193227 (70 d. C.): Dule, soldado de la legión II Adiutrix. Como testigo de su licenciamiento honroso encontramos a Publius Lucretius Apulus de la cohorte IX Pretoriana. Ambos comparten el mismo origen.

- CIL XVI 12 = AE 191210 = AE 191344 = AE 192054 (71 d. C.) : El centurión Hezbenus Sappaeus y su hijo Doles, pertenecientes a la flota de Misenum, licenciados en un diploma militar entre cuyos testigos firmantes aparece Caius Sallustius Crescens de la cohorte IIII Pretoriana. Puesto que todos los testigos son originarios de Philippi es muy probable que fuese éste el vínculo que los unía.

- CIL VI 2545 = CIL XI 101, 2 = ILS 2064 (100-180 d. C.): Sextus Pufius Quartus de la cohorte IV Pretoriana, cuyo epígrafe lo dedica Alliator Ories, stator del emperador y conciudadano suyo.

- CIL VI 2970: Caius Calvius Sabinus de la cohorte III de los vigiles, cuya inscripción fue dedicada por su hermano, Calvius lustus de la cohorte I Pretoriana.

\section{RESTO DE RELACIONES INTERPERSONALES}

Naturalmente, existen relaciones entre el resto de soldados de la guarnición urbana que no atañen en modo alguno a los soldados pretorianos. Veamos los casos:

Durante el siglo II d. C. aparecen relaciones entre soldados de las cohortes urbanas y el resto de tropas ejemplificados en el caso de Caius Monnenius Secundus de la cohorte XIIII Urbana, cuyo epígrafe está dedicado por su hermano Lucius Monnenius Secundus de la cohorte IIII de los vigiles (AE 1948 68). Así como en el caso de Titus Flavius lulius, sesquiplicarius de los jinetes singulares del emperador, cuyo heredero y amigo, Titus Flavius Florentinus de la cohorte XI Urbana, le dedica la inscripción (CIL VI 3253 = Denkm 114), en una fecha imprecisa de los siglos I ó II d. C.

En el siglo III d. C. tenemos a Caius Marius Gemellinus, veterano de los jinetes singulares de nuestro emperador, cuyo epígrafe está dedicado por Marius Marcellinus, soldado de la legión II Parthica (CIL VI 3277 = Denmk 736). En la misma situación encontramos a un jinete singularis desconocido cuyo epígrafe fue dedicado por Publius Aelius de la legión II Parthica (CIL VI 37258a = Denkm 547).

Por último, curioso es el caso de Titus Aurelius Leo (CIL VI $2992=$ Denkm 734), quien no era militar, pero aparece tanto como dedicante de la inscripción de 
Titus Aurelius Salutaris, veterano de los jinetes singulares de nuestros emperadores y patrono suyo, como del epígrafe de Severinus, soldado de la cohorte VI de los vigiles, de quien era hermano. Dado el estado fragmentario del epígrafe es posible que el mismo Severinus fuese liberto de Titus Aurelius Salutaris.

\section{CONCLUSIONES}

El recuento epigráfico resulta, ciertamente, escaso, especialmente si tenemos en cuenta que existen en torno a 3000 epígrafes realizados por la guarnición urbana. Sin embargo, podemos alcanzar algunas conclusiones.

La vinculación entre soldados pretorianos de distintas cohortes por sangre es menor y existe sólo con anterioridad a Septimio Severo, lo cual refuerza la teoría de que los parientes servían generalmente en la misma cohorte, de lo cual hay diversos ejemplos ${ }^{5}$.

Las relaciones de amistad entre soldados pretorianos de distinta cohorte se constatan a partir de la segunda mitad del siglo I d. C. (CIL XI 6348). En la mayoría de los casos el vínculo de unión es un origen común. Hay que destacar que este fenómeno se da sobre todo entre los provinciales (CIL VI 2520, AE 1924107 y quizá CIL VI 2545 = CIL XI 101, 2 = ILS 2064), alejados de su hogar, que debían de encontrar un fuerte apoyo en sus conciudadanos. Esta atracción entre soldados pretorianos conterráneos y el vínculo que los une se reforzará con el tiempo, especialmente a partir de la reforma severiana. Hasta este momento la mayoría de los pretorianos procedían de tierras italianas y nada otorgaba una identidad diferenciada a un pretoriano sobre otro, obviando un pequeño número de extraitálicos, pero la reforma del pretorio introdujo a un número muy alto de soldados de una gran variedad de procedencias, principalmente la frontera del Danubio.

Desde entonces se concentran en Roma un número muy alto de soldados extraitálicos procedentes de la misma provincia, con una marcada personalidad que contrasta con la de la plebe urbana ${ }^{6}$. Esto, junto con el culto a dioses locales lleva a los soldados pretorianos a formar grupos de amistad mucho más amplios y frecuentes que en el siglo anterior (CIL VI 2732, CIL VI $2799=$ CIL VI $32543=$ ILS 2094, CIL VI 2822 = CIL VI $3902=$ CIL VI $3903=$ CIL VI 32550a-b = AE 189418 y CIL VI $2821=$ CIL VI $32551=$ ILS 2096, CIL VI 2831= CIL VI $2852=$ CIL VI 32557, CIL VI $2807=$ CIL VI 32582 = ILS 4068, CIL VI 32571 y probablemente AE 198350 y CIL VI 2742). Ciertamente, casi todas las relaciones de amistad entre soldados pretorianos de distinta cohorte en el siglo III d. C. responden a este esquema. Por tanto, la vecindad pasó a ser un elemento importante a la hora de escoger con quienes relacionarse.

5 AE 2004 309, AE 2004 312, CIL VI 2491, CIL XI 6350 = ILS 9066, CIL VI 2653, CIL VI 2718, AE 1924 107, CIL VI 2692, CIL VI 2753, CIL VI 32671, CIL X 1755 = Inscrlt I 1, no $146=$ AE 1992 85, CIL VI 2600, CIL VI 3894 = CIL VI 32690, CIL VI 2616 ó CIL VI 2756.

6 Dión Cas. LXXIV, 2, 4-6. 
En lo que respecta a la relación entre los pretorianos y los urbaniciani nos sorprende sobremanera la casi total ausencia de relaciones entre ambas tropas, limitándose prácticamente a los lazos de sangre e interrumpiéndose durante el gobierno tras el gobierno de Septimio Severo. La causa obvia de esta interrupción es que los pretorianos procedían ahora de fuera de Italia mientras que los urbaniciani seguían siendo reclutados dentro de la península.

Dadas las múltiples características en común entre ambas tropas ${ }^{7}$ y la efusiva reacción de los soldados de las cohortes urbanas a favor de los pretorianos, a los que literalmente definen como «parientes» durante el ascenso de Claudio ${ }^{8}$, nos esperábamos una unión mucho más fuerte y frecuente entre ambos. Más aún si tenemos presente que, compartiendo las cohortes pretorianas y urbanas el espacio común de los Castra Praetoria, el contacto debía de ser frecuente.

Por lo tanto, el uso del mismo espacio no bastaba para ligar a unos soldados con otros, ¿por qué, entonces, soldados pretorianos procedentes de distinta cohorte aparecen conectados entre sí con tanta frecuencia? Por dos motivos. En primer lugar la identidad como grupo, ya que aunque oficialmente las cohortes pretorianas eran unidades independientes, de facto actuaban y pensaban como una única entidad. En segundo lugar está el hecho de que cada cohorte pretoriana compartía funciones con las demás, es decir, no sólo ocupaba el mismo lugar de habitación, sino también el mismo lugar y responsabilidades en el palacio y el campo de batalla, diferenciándose de todos los demás pero no entre sí.

Por ello, no existen relaciones de amistad entre los soldados de las distintas cohortes de vigiles. Compartían funciones en efecto, pero cada cohorte tenía asignada una jurisdicción concreta dentro de la Urbe y no entraba en la de otra salvo en los casos más graves. Compartían la misión pero no el lugar de acantonamiento.

Los periodos intensos de campañas militares parecen haber sido uno de los principales factores que llevan a los soldados de la guarnición urbana a simpatizar entre sí. De esta manera, es a partir del siglo III d. C., más de ochenta años después de su creación, cuando los equites singulares Augusti aparecen compartiendo epígrafes con los pretorianos. Es decir, después de que ambos cuerpos integren el ejército central de maniobra y marchen juntos a cada guerra ${ }^{9}$.

Los vínculos entre los pretorianos y la legión II Phartica hemos de situarlos exactamente en el mismo contexto, así como las relaciones entre los singulares y los albanoi (CIL VI 3277 = Denmk 736 y CIL VI 37258a = Denkm 547).

7 Las cohortes urbanas eran originalmente cohortes pretorianas con una numeración correlativa con respecto a la de aquellas; los urbaniciani pueden ser transferidos, por merito, a las cohortes pretorianas; los veteranos de ambas tropas se mencionan juntos en los diplomas militares como pertenecientes al pretorio y existen cementerios comunes para ambas tropas.

8 F. J. Bell. lud., II, 10.

9 También hay que añadir que desde la reforma severiana era mucho más probable que pretorianos y singulares procediesen de las mismas provincias lo que ayudaría a estrechar lazos. En algunos de los casos (ILS $9069=$ AE $189433=$ Denkm 747, CIL VI $32680=$ CIL X $3582=$ Denmak 750, AE $195479=$ Denmk 530 y AE 198349 = Denmk 608) estaban incluso emparentados. 
Las campañas militares creaban una identidad de grupo entre los soldados movilizados, un «nosotros = romanos» frente a un «ellos = bárbaros», que hacía olvidar cualquier rencilla y estrechaba las relaciones a nivel general entre las distintas unidades militares. Esta es la causa de que aparezcan ahora dedicaciones de oficiales del mismo rango procedentes de distintas cohortes pretorianas (CIL VI 2385b 19 = CIL VI 2385b $24=\mathrm{CIL}$ VI 2388a $1=\mathrm{CIL}$ VI 2388a $2=\mathrm{CIL}$ VI 2388a $3=$ CIL VI 2388a 4 = CIL VI $3797=$ CIL VI $3798=$ CIL VI $31491=$ CIL VI 32538).

Otra buena prueba de ello son dos diplomas militares inmediatamente posteriores a la guerra civil del año 69 d. C. (CIL XVI $10=$ AE 193227 y CIL XVI $12=$ AE 191210 = AE 191344 = AE 1920 54) ambos de antiguos marinos, uno de ellos trasladado a la legión II Adiutrix. En los dos casos estamos ante amistades surgidas durante la guerra. Parece que los licenciados escogieron como testigos de los diplomas a aquellas de sus amistades, tanto civiles como militares, procedentes de su misma ciudad de nacimiento.

La buena disposición creada en el ambiente bélico desaparecía dentro de la Urbe. No es necesario decir que el emperador no deseaba una dependencia fuerte entre las unidades de la guarnición urbana. El trato debía ser cordial, pero nada más. De no ser así, el emperador se arriesgaba a que la sedición de una de las tropas de más peso arrastrase tras de sí a las demás. Baste mencionar, como ya hicimos, que fue una situación idéntica la que terminó de aupar a Claudio, y por tanto, una situación idéntica podría haber aupado a la púrpura a un candidato en perjuicio del emperador legítimo.

Ejemplo de esto aparecen en la literatura clásica, en donde encontramos a urbaniciani y a singulares luchando en las calles durante el gobierno de Commodo ${ }^{10}$. Ménard opina que este es el primer ejemplo de enfrentamientos entre soldados de la guarnición urbana ${ }^{11}$, culminando en el año 238 d. C. cuando los pretorianos, realmente atemorizados ante los auxiliares germanos, asesinen a Máximo y Balbino para no ser reemplazados ${ }^{12}$.

De hecho, ningún epígrafe muestra relación alguna entre los pretorianos y cualquier cuerpo de germanos establecido en Roma, y sólo uno menciona una amistad entre un urbanicianus y un eques singularis.

La teoría no parece descabellada. Hacía unos pocos años se había producido una ruptura importante como consecuencia de las guerras sufridas durante el gobierno de Marco Aurelio. La extrema situación obligó a desplazar a las cohortes pretorianas y a los singulares como nunca antes, adelantando ya las medidas severianas.

Se produce una importante dicotomía entre las unidades militares de la guarnición urbana: aquellos que pasan largas temporadas fuera de la Ciudad en cam-

\footnotetext{
10 Her. I, 12, 5-9.

11 Ménard, H., 2004: 50.

12 Her. VIII, 7, 7-8 y 8, 1-6.
} 
paña (pretorianos y singulares) y aquellas que no salen de la Ciudad o de Italia (cohortes urbanas y vigiles).

Varios factores debieron ayudar al distanciamiento desde este momento:

- Ios urbaniciani deben asumir funciones policiales que antes correspondían a los pretorianos, mientras éstos se hallaban fuera y adquieren un rol más relevante en la posible defensa de Italia. Así su papel en la Urbe se vuelve más poderoso, pero siguen quedando en segundo plano frente a las cohortes pretorianas.

- Las cohortes urbanas abandonan los Castra Praetoria durante el gobierno de Commodo ${ }^{13}$. Con la creación de los Castra Urbana quedaba rota toda posibilidad de crear lazos entre las cohortes urbanas y los singulares.

- Hasta época severiana los soldados de las cohortes urbanas y pretorianas proceden de los mismos lugares dentro de la península itálica, pero desde Septimio Severo los pretorianos proceden de las mismas regiones que los singulares. De esta forma los pretorianos tienen cada vez menos cosas en común con los urbaniciani y más con los singulares. De hecho, todas las relaciones de amistad y los lazos de sangre entre soldados de estos dos cuerpos se circunscriben al siglo III d. C.

El último ejemplo de esta separación lo encontramos bajo el gobierno de Majencio, emperador por el cual pelean tanto pretorianos como equites singulares, sufriendo ambas unidades la misma suerte tras su derrota ${ }^{14}$. En cambio las cohortes urbanas sobrevivieron al episodio y continuaron guardando la ciudad de Roma durante el siglo IV d. C..$^{15}$, lo que viene a indicar que no secundaron a pretorianos y singulares en su apoyo a Majencio. Las cohortes urbanas tenían pues intereses particulares y bien diferenciados.

\section{BIBLIOGRAFÍA}

CLAUSS, M. (1973): «Zur Datierung der Stadtrömischen Inschriften: Tituli Militum Praetorianorum», Epigraphica XXXV, pp. 55-95.

CEÑAL MARTíNEZ, H. (2009): La guardia pretoriana. Composición, funciones e historia (tesis doctoral s. p.), Universidad de Oviedo.

DURRY, M. (1968²): Les cohortes pretoriennes, París (Éditions E. de Boccard).

FREIS, H. (1967): Die Cohortes Urbanae, Colonia (Ed. Rheinland Verlag).

MÉNARD, H. (2004) : Maintenir l'ordre à Rome (Ile-IVe AP. J.-C.), Seyssel (ed. ChampVallon).

13 Sablayrolles, R., 2001: 140.

14 Speidel, M. P., 1986: 255-256.

15 Freis, H., 1967: 19. 
PANCIERA, S. (2004): «Altri pretoriani a Roma. Nuove iscrizioni e vecchie domande», Les unités de la garnison de urbaine et leur épigraphie à Rome et hors de Rome. Cahiers de Centre G. Glotz 15, pp. 281-316.

RICCI, C. (1994): Soldati delle milizie urbane fuori di Roma. La documentazione epigrafica, Roma (Edizioni Quasar)

SABLAYROLLES, R. (2001): «La rue, le soldat et le pouvoir: la garnison de Rome de Caesar à Pertinax», Pallas 55, pp. 127-153.

SPEIDEL, M. P. (1965): Die Equites Singulares Augusti. Begleittruppe der römischen kaiser des zweiten und dritten jahrhunderts, Bonn (ed. Rudolf Habelt Verlag).

SPEIDEL, M. P. (1986): «Maxentius and his equites singulares», Classical Antiquity 5, pp. 253-263. 
